Article

\title{
Functionalized Graphene Quantum Dots Modulate Malignancy of Glioblastoma Multiforme by Downregulating Neurospheres Formation
}

\author{
Giordano Perini ${ }^{1,2}$ (1), Valentina Palmieri ${ }^{1,2,3, *}$, Gabriele Ciasca ${ }^{1,2}$, Aniello Primiano ${ }^{2,4}$, Jacopo Gervasoni ${ }^{2,4}$, \\ Marco De Spirito ${ }^{1,2}$ and Massimiliano Papi ${ }^{1,2, * \mathbb{D}}$ \\ 1 Dipartimento di Neuroscienze, Università Cattolica del Sacro Cuore, 00168 Rome, Italy; \\ giordano.perini@unicatt.it (G.P.); gabriele.ciasca@unicatt.it (G.C.); marco.despirito@unicatt.it (M.D.S.) \\ 2 Fondazione Policlinico Universitario A. Gemelli IRCCS, 00168 Rome, Italy; aniello.primiano@unicatt.it (A.P.); \\ jacopo.gervasoni@policlinicogemelli.it (J.G.) \\ 3 Institute for Complex Systems, National Research Council (ISC-CNR), Via dei Taurini 19, 00185 Rome, Italy \\ 4 Dipartimento di Scienze Biotecnologiche di Base, Cliniche Intensivologiche e Perioperatorie, \\ Università Cattolica del Sacro Cuore, 00185 Rome, Italy \\ * Correspondence: valentina.palmieri@cnr.it (V.P.); massimiliano.papi@unicatt.it (M.P.)
}

check for updates

Citation: Perini, G.; Palmieri, V.; Ciasca, G.; Primiano, A.; Gervasoni, J.; De Spirito, M.; Papi, M.

Functionalized Graphene Quantum Dots Modulate Malignancy of Glioblastoma Multiforme by Downregulating Neurospheres Formation. C 2021, 7, 4. https:// doi.org/10.3390/c7010004

Received: 27 November 2020 Accepted: 31 December 2020 Published: 5 January 2021

Publisher's Note: MDPI stays neutral with regard to jurisdictional clai$\mathrm{ms}$ in published maps and institutional affiliations.

Copyright: (C) 2021 by the authors. Licensee MDPI, Basel, Switzerland. This article is an open access article distributed under the terms and conditions of the Creative Commons Attribution (CC BY) license (https:// creativecommons.org/licenses/by/ $4.0 /)$.

\begin{abstract}
Glioblastoma multiforme (GBM) is the most aggressive brain cancer. We previously demonstrated the effect of biocompatible surface functionalized graphene quantum dots (GQDs) on GBM cells as chemotherapy enhancers in combination with the antitumor drug doxorubicin (Dox). However, traditional two-dimensional cultures could not represent a reliable model of tumor behavior. In this work, we investigated the effect of carboxylated (COOH-GQDs), aminated $\left(\mathrm{NH}_{2}-\mathrm{GQDs}\right)$ and unfunctionalized GQDs on a three-dimensional model of neurospheres. Neurospheres are clusters of GBM cells, which formation is driven by the presence of a stem subpopulation involved in cancer malignancy. Tumor recurrence after surgical resection, chemotherapy and radiotherapy indeed depends on the presence of cancer cells with stem properties. We measured a significant reduction in number and size of neurospheres after two weeks of monitoring in the presence of COOH-GQDs and GQDs. Previous works pointed out how variations of membrane fluidity could affect membrane stability and cell-to-cell interactions, thus influencing cell clustering. Therefore, we measured changes in membrane fluidity after administration of GQDs. We found that COOH-GQDs and GQDs significantly increased membrane fluidity with respect to the treatment with $\mathrm{NH}_{2}$-GQDs or compared to untreated cells. Shifts in the phase of phospholipid bilayer were in accordance with the negative surface net charge of GQDs. We depicted a strong correlation between negatively charged GQDs-induced increase in membrane fluidity and the downregulation of neurospheres formation. Our results indicate that COOH-GQDs and GQDs significantly modulate tumor malignancy by increasing fluidity of cell membrane, with a consequent inhibition of cell-to-cell interaction.
\end{abstract}

Keywords: glioblastoma; graphene quantum dots; neurospheres; nanomedicine

\section{Introduction}

Among gliomas, glioblastoma multiforme (GBM) is known to be the most common, aggressive and lethal. The standard GBM treatment includes surgical resection, radiotherapy and chemotherapy [1,2]. However, at the best circumstances, the median of survival is only extended from two months to one year. Several approaches are under investigation for fighting GBM, but a clinical translation has not yet occurred, mainly due to lack of reliable experimental models capable of faithfully reproducing tumor complexity [3-5].

In our previous studies, we investigated the effect of graphene quantum dots (GQDs) functionalized with different surface groups on glioblastoma multiforme (GBM) cell line [6-8]. We demonstrated the effectiveness of GQDs and carboxylated GQDs (COOHGQDs) as enhancing agents along with the antitumor drug doxorubicin (Dox). We found a 
correlation between the synergistic effect of GQDs and Dox with the surface net charge of the nanoparticles. However, traditional two-dimensional cultures could not represent a reliable model of tumor behavior.

Here, we tested the effect of functionalized GQDs on a three-dimensional culture model of neurospheres. Neurospheres grow in semi-suspension conditions, and their formation is a relevant indicator of the malignancy and invasiveness of the tumor itself [9]. At first, we evaluated the biocompatibility of aminated ( $\mathrm{NH}_{2}$-GQDs) $\mathrm{COOH}-\mathrm{GQDs}$ and unfunctionalized GQDs on GBM in a timespan of two weeks. We then moved to monitor the formation of neurospheres by acquiring images at different timepoints. We found a strong inhibition of spheres formation after the treatment with GQDs and COOH-GQDs, in terms of number and size of neurospheres. We also detected a reduction in the number of connections between neurospheres, by developing an algorithm based on spatial networking. The development of connections among neurospheres is relevant for the trophism of GBM, similarly to astrocytes endfeet cell-to-cell interactions [10].

Neurospheres formation is strictly dependent to the presence of a tumor stem subpopulation, which has the capability to regrow after surgical resection and standard radiochemotherapy [11]. Therefore, the inhibition of formation of neurospheres has a great clinical relevance to downregulate tumorigenesis along with recurrent GBM phenomena, possibly improving prognosis and quality of life of patients.

\section{Results and Discussion}

\subsection{Characterization of GQDs}

GQDs, $\mathrm{NH}_{2}$-GQDs and $\mathrm{COOH}-\mathrm{GQDs}$ were characterized by transmission electron microscopy (TEM), fluorescence spectroscopy and attenuated total reflection Fourier transform infrared (ATR-FTIR) spectroscopy. TEM imaging experiments were carried out on all GQDs, and images revealed particles smaller than $10 \mathrm{~nm}$, in accordance with manufacturers' specifications (Figure 1A-C). The IR spectra of $\mathrm{NH}_{2}$-GQDs showed a band around $\sim 3200 \mathrm{~cm}^{-1}$ that was associated with the $\mathrm{N}-\mathrm{H}$ stretching vibration of amine groups (Figure 1D, circle 1) [12]. The band at $1656 \mathrm{~cm}^{-1}$ corresponded to amide I (the $-\mathrm{C}=\mathrm{O}$ stretch) and at $1536 \mathrm{~cm}^{-1}$ was assigned to amide II (the $-\mathrm{C}-\mathrm{N}$ stretch and $-\mathrm{C}-\mathrm{N}-\mathrm{H}$ deformation, circle 2) [13]. Finally, a band around $1403 \mathrm{~cm}^{-1}$ was due to $\mathrm{C}-\mathrm{N}$ absorption. The infrared (IR) spectra of $\mathrm{COOH}-\mathrm{GQDs}$ showed characteristic IR frequencies of the carboxylic acid group, a broad band between $3000-2800 \mathrm{~cm}^{-1}$ for the $\mathrm{O}-\mathrm{H}$ stretch (Figure 1E, circle 1) and a very strong band at $1705 \mathrm{~cm}^{-1}$ for the $\mathrm{C}=\mathrm{O}$ stretch (Figure 1E, circle 2). The IR spectra of GQDs showed a band at $3333 \mathrm{~cm}^{-1}$ due to $\mathrm{O}-\mathrm{H}$ stretching vibrations (Figure $1 \mathrm{~F}$, circle 1), at $1613 \mathrm{~cm}^{-1}$ due to $\mathrm{C}=\mathrm{C}$ bond (Figure $1 \mathrm{~F}$, circle 2) and $1260 \mathrm{~cm}^{-1}$ epoxide $\mathrm{C}-\mathrm{O}$ stretching vibrations (Figure 1F, circle 3). Fluorescence spectroscopy was performed in spectral scanning (Figure 1G-I). Fluorescence spectra were acquired by exciting in a range from 360 to $500 \mathrm{~nm}$. All of GQDs revealed an excitation-dependent behavior. $\mathrm{NH}_{2}-\mathrm{GQDs}$ revealed an emission peak at $420 \mathrm{~nm}$ when excited at $320 \mathrm{~nm}$ (Figure 1G). Fluorescence intensity peak resulting from amination was in accordance with the characteristic blue emission at $438 \mathrm{~nm}$, which has been previously reported [14]. COOH-GQDs showed a peak at $450 \mathrm{~nm}$ when excited at $320 \mathrm{~nm}$ (Figure 1H). GQDs depicted an emission peak at $540 \mathrm{~nm}$ when excited at $480 \mathrm{~nm}$ (Figure 1I). Emission peak of COOH-GQDs has already been measured by Yuan, Liu and colleagues [15]. However, in their work, maximum intensity of COOH-GQDs was obtained when exciting at $400 \mathrm{~nm}$. Optical properties of GQDs are known to strongly depend on their surface functional groups. The presence of oxygen or nitrogen species causes blue shifts in the photoluminescent emission peaks due to formation of smaller $\mathrm{sp}^{2}$ aromatic clusters, which contribute to increase the energy gap between valence and conduction band [8]. Fluorescence intensity of $\mathrm{NH}_{2}$-GQDs was higher than that of $\mathrm{COOH}-\mathrm{GQD}$. Nitrogen groups carrying a lone pair tend to increase quantum yield indeed, due to $n-\pi^{*}$ energy transitions, thus resulting in an increased fluorescence intensity [16]. 

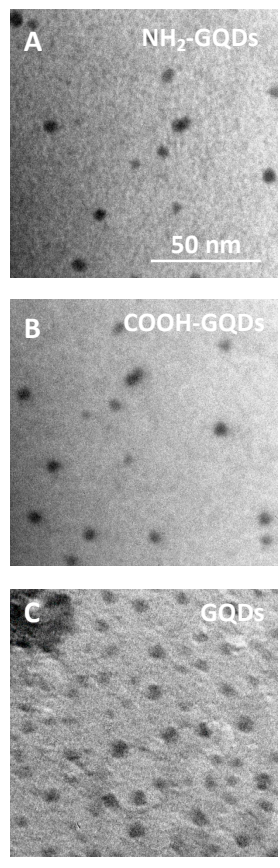
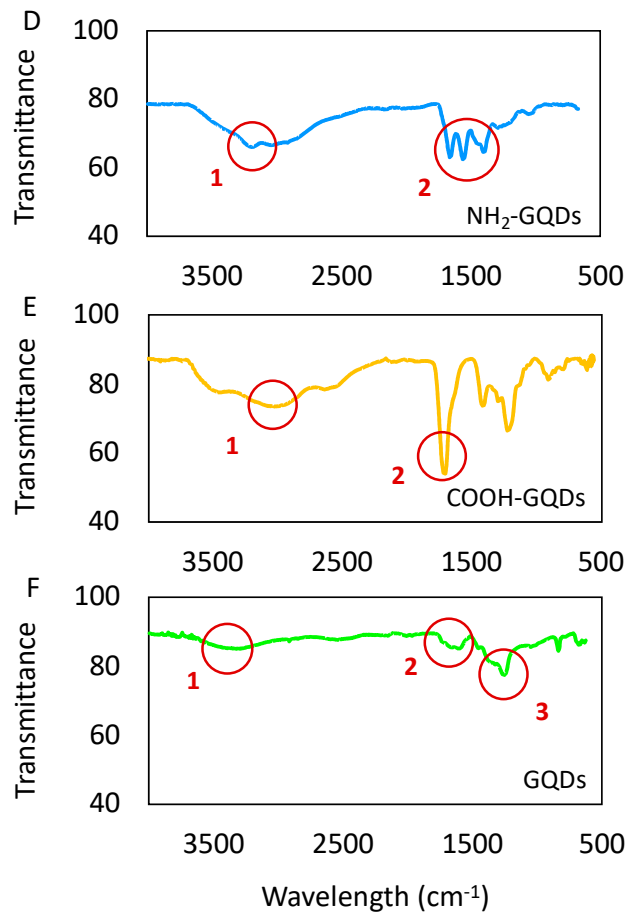
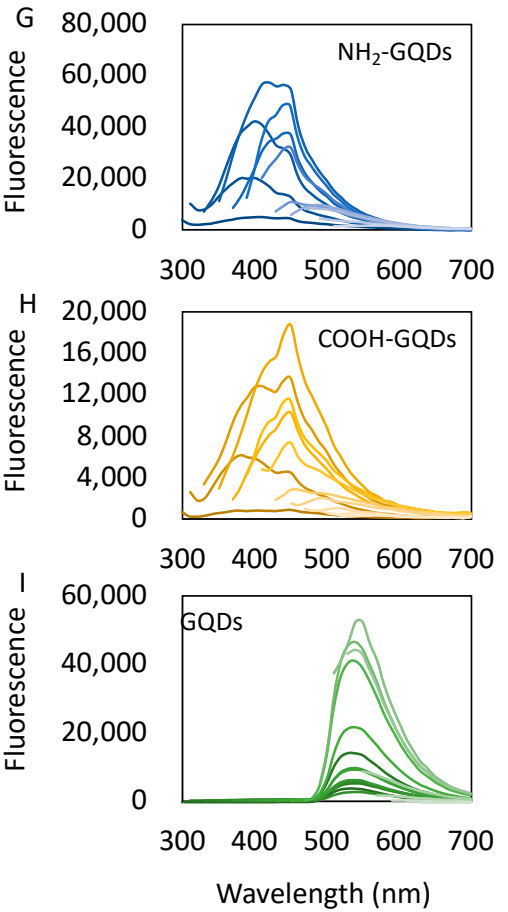

Figure 1. Characterization of graphene quantum dots (GQDs). (A-C) Transmission electron microscopy of aminated GDQs ( $\mathrm{NH}_{2}-\mathrm{GQDs}$ ), carboxylated GDQs (COOH-GQDs) and GQDs, respectively. (D-F) Fourier transform infrared (FTIR) spectroscopy of GQDs. The numbered red circles highlight specific regions of the spectra typical of the respective functional groups. Circle 1,2 of Figure 1D indicates stretching of amines and amide II, respectively. Circle 1,2 of Figure 1E indicates $\mathrm{O}-\mathrm{H}$ and $\mathrm{C}=\mathrm{O}$ stretching respectively. Circle $1-3$ of Figure $1 \mathrm{~F}$ indicates stretching vibration of $\mathrm{O}-\mathrm{H}, \mathrm{C}-\mathrm{C}$ and $\mathrm{C}-\mathrm{O}$. (G-I) Fluorescence spectra of $\mathrm{NH}_{2}$-GQDs, $\mathrm{COOH}-\mathrm{GQDs}$ and GQDs, respectively, at excitation wavelengths ranging from 260 to $500 \mathrm{~nm}$.

While microscopic characterization pointed out the uniform size of the nanoparticles, IR spectroscopy and fluorescence spectroscopy highlighted their diverse specific surface chemical functionalization.

\subsection{Biocompatibility of GQDs}

We performed biocompatibility tests on glioblastoma cells to exclude the contribution of a possible toxicity of GQDs in the analysis of neurospheres' formation. Biocompatibility of GQDs was assessed on U87MG human glioblastoma cell line by 3-(4,5-dimethylthiazol2-yl)-2,5-diphenyltetrazolium bromide (MTT) test after the treatment with 50, 100 or $200 \mu \mathrm{g} / \mathrm{mL}$ of GQDs. Formation of neurospheres was monitored for 14 days. Therefore, both acute response and long-time exposure to GQDs was measured, from $24 \mathrm{~h}$ up to 14 days after nanoparticles' administration. Cell viability after the treatment with GQDs, $\mathrm{NH}_{2}$-GQDs and $\mathrm{COOH}-\mathrm{GQD}$ is reported normalized by control (untreated) cells (Figure 2A-C). No significant differences were depicted by biocompatibility measurements for all GQDs, even at the highest tested concentration $(200 \mu \mathrm{g} / \mathrm{mL})$. U87MG cells showed a viability of $90 \% \pm 6.2$ and $9.6 \%$ after 14 days of treatment with $\mathrm{NH}_{2}-\mathrm{GQDs}$ at 50 and $100 \mu \mathrm{g} / \mathrm{mL}$ respectively, while $200 \mu \mathrm{g} / \mathrm{mL}$ of $\mathrm{NH}_{2}$-GQDs exerted a reduction in viability up to $86 \% \pm 5.7 \%$ (Figure $2 \mathrm{~A}$ ). MTT test did not point out toxicity related to $\mathrm{COOH}-\mathrm{GQDs}$ administration as well. After 14 days, U87MG maintained a viability of 98,95 and $89 \% \pm 1.6,3.5$ and $8.7 \%$, respectively, at 50, 100 and $200 \mu \mathrm{g} / \mathrm{mL}$ (Figure 2B). Unfunctionalized GQDs did not induce toxicity on GBM as well. Viability after two weeks remained at the biocompatible levels of 94,97 and $93 \% \pm 6.2,9.3$ and $5.1 \%$, respectively, at 50, 100 and $200 \mu \mathrm{g} / \mathrm{mL}$ (Figure 2C). We already reported the high biocompatibility of surface-functionalized GQDs [6-8]. Our data are in accordance with most of the current 
literature. Fasbender and colleagues indeed reported high viability of human red blood cells even after administration of GQDs at $500 \mu \mathrm{g} / \mathrm{mL}$ [17]. In their work, they investigated in vitro exposure of GQDs, which resulted in a time- and concentration-dependent cellular uptake. Intracellular uptake of quantum dots was not related to any evident toxicity. The uptake was also measured, in the same work, on lymphocytes, granulocytes and monocytes, which had a result higher in the latter when compared to lymphocytes. They stated that their results intrinsically point out a different behavior in membrane permeability, depending on cell type. Shang and colleagues measured uptake of GQDs within human neural stem cells, along with relative metabolic activity [18]. Viability tests outlined high biocompatibility of neural stem cells even at relatively high concentrations of nanoparticles $(250 \mu \mathrm{g} / \mathrm{mL})$. They also measured quantum dots retention inside cells over time, pointing out relative reduction in fluorescence intensity within 3 days. Surface functionalization of GQDs resulted in different toxic or nontoxic outcomes. Nurunnabi and colleagues highlighted low cytotoxicity of carboxylated GQDs at concentrations ranging from 0 to $250 \mu \mathrm{g} / \mathrm{mL}$, while a decrease in metabolic activity was present after their administration at $500 \mu \mathrm{g} / \mathrm{mL}$, along with an increase in lactate dehydrogenase production, in KB cell line. A different behavior was measured in MDA-MB231, and in A549 cells, in which cell viability was around $80 \%$ even at the highest tested concentration of GQDs [19]. Yuan and coworkers investigated the effect on A549 and C6 cell lines of three different surface chemistries: carboxylation, amination and functionalization with dimethylformamide (DMF). In all cases, they measured a slight reduction in cell viability as well as a very low mortality [15]. Li and colleagues described in vivo response and toxic effects on C. elegans to long exposure to graphene-based materials, by considering changes in specific physiological parameters such as head trashes and bending angles [20]. Under sublethal exposure condition the three materials, namely pristine graphene, graphene oxide and GQDs, had similar effects on behavior and neurotoxicity on nematodes. They found that GQDs indeed possess relatively low lethality and neural toxicity even at the highest-tested concentration of $100 \mathrm{mg} \mathrm{L}^{-1}$. Our cytotoxicity tests highlight the great biocompatibility of GQDs and surface functionalized GQDs even after long-time exposure.

\subsection{Formation of Neurospheres}

Once assessed viability on U87MG cells after 14 days of exposure to GQDs, we moved to evaluate the effect of the graphene nanoparticles in the formation of neurospheres. Figure 3 depicts the formation of neurospheres after the treatment with $200 \mu \mathrm{g} / \mathrm{mL}$ of $\mathrm{NH}_{2}$-GQDs, COOH-GQDs and GQDs. Representative images display how $\mathrm{NH}_{2}$-GQDs did not exert an effect on reduction in neurospheres formation, which remained consistently similar to that of control (untreated) GBM (Figure 3A). COOH-GQDs and GQDs, on the contrary, significantly affected the formation of neurospheres, by inhibiting their growth during the time course of the experiment (Figure 3A). We then measured the number and size of neurospheres formed over two weeks in the three conditions. We used an image analysis method that considered a neurosphere formed above 150 pixels, which corresponded to a sphere with a radius of $\sim 50 \mu \mathrm{m}$. The number of neurospheres formed per $\mathrm{cm}^{2}$ this way is reported in Figure 3B. Cells treated with $\mathrm{NH}_{2}-\mathrm{GQDs}$ reached a density of $10.5 \pm 3.5$ neurospheres $/ \mathrm{cm}^{2}$, which were results similar to those of control U87MG cells (11.5 \pm 1 neurospheres $/ \mathrm{cm}^{2}$, Figure $\left.3 \mathrm{~B}\right)$. All cultures showed a plateau in their growth after one week, probably due to confluency effect. COOH-GQDs, on the contrary, significantly reduced the number of neurospheres $\left(0.92 \pm 0.45\right.$ neurospheres $/ \mathrm{cm}^{2}$, Figure 3B). GQDs influenced the number of neurospheres as well, which decreased at $1.01 \pm 0.6$ neurospheres $/ \mathrm{cm}^{2}$ (Figure 3B). As a consequence, the size of clusters was influenced by GQDs' administration. While the average size was $279 \pm 40$ and $265 \pm 34$ after 14 days from the administration of GQDs in control cells and cells treated with $\mathrm{NH}_{2}-$ GQDs, respectively, COOH-GQDs and GQDs significantly inhibited formation of clusters, allowing cells to reach an average size of $79 \pm 24$ and $78 \pm 29$ pixels, respectively. 


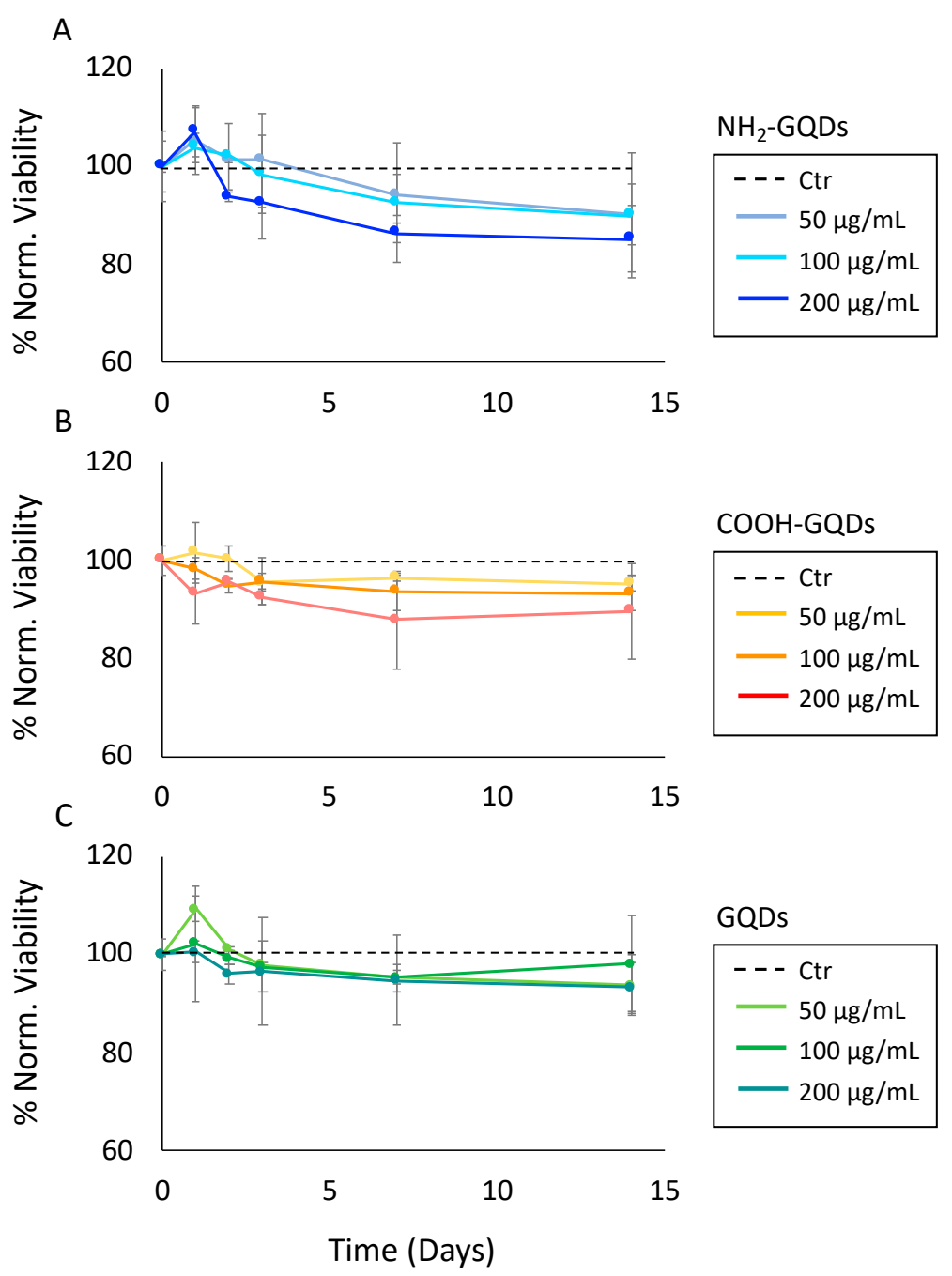

Figure 2. Biocompatibility of glioblastoma multiforme (GBM) after the treatment with GQDs in two weeks. (A) Biocompatibility of U87 glioblastoma cells after the treatment with 50, 100 or $200 \mu \mathrm{g} / \mathrm{mL}$ of $\mathrm{NH}_{2}$-GQDs. (B) Biocompatibility of U87 glioblastoma cells after the treatment with 50, 100 or 200 $\mu \mathrm{g} / \mathrm{mL}$ of COOH-GQDs. (C) Biocompatibility of U87 GBM cells after the treatment with 50, 100 or $200 \mu \mathrm{g} / \mathrm{mL}$ of GQDs.

Neurospheres formation is strictly related to the malignancy of GBM. Even after aggressive treatment that eliminates most of the tumor load, this tumor recurs in an average of 6.9 months after initial treatment [21]. The capability of GBM to regrow after surgical resection and radiochemotherapy implies that there is a subpopulation of cells with proliferative activity. Neurosphere cultures of GBM are heterogeneous, like the tumor itself, and only a fraction of cells is capable of sphere formation. These stem cells have been classified as neurosphere-initiating cells [22]. The identification of stem cells in brain tumors provides a powerful tool to investigate the tumorigenic process in the CNS and to develop therapies targeted to these cells. Cancer stem cells responsible for forming neurospheres and initiating tumor development have shown to overexpress several biomarkers, such as CD44, CD133 and GFAP. CD133, in particular, is largely used as marker in several tumors, including GBM. Originally, it was identified as a surface antigen expressed on hematopoietic stem cells [23]. GFAP, a member of the cytoskeletal protein family, is widely expressed in astroglial cells and in neural stem cells [24]. However, it has also been demonstrated that it is overexpressed in astroglial tumors, such as astrocytoma and GBM [25]. Steponkiene and colleagues demonstrated how treatment with QDs on GBM cells affected the subpopulation of most malignant stem cells [26]. In their work, stem subpopulations were divided 
depending on their expression of CD44 and CD133. When compared with the control untreated cells, the cells treated with QDs contained 1.4 times more of the $\mathrm{CD} 44^{\text {high }} \mathrm{CD} 133^{\text {low }} /$ cells (39\% versus $28 \%$ in the control) and 1.3 times less of the CD $44^{\text {high }} \mathrm{CD} 133^{\text {high }}$ cells ( $54 \%$ versus $72 \%$ in the control). The percentage of CD $44^{\text {low } /-C D 133^{\text {low } /-} \text { cells significantly }}$ increased after the treatment. These results are in accordance with our experimental data and suggest that GQDs-mediated alterations in the expression of stem cells biomarkers could be due to changes in the CD44/ CD133 subpopulations.
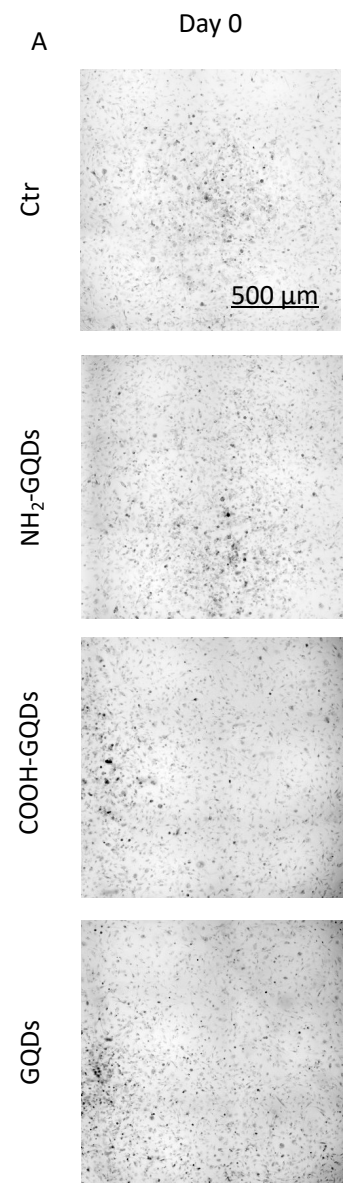

$$
\text { Day } 7
$$
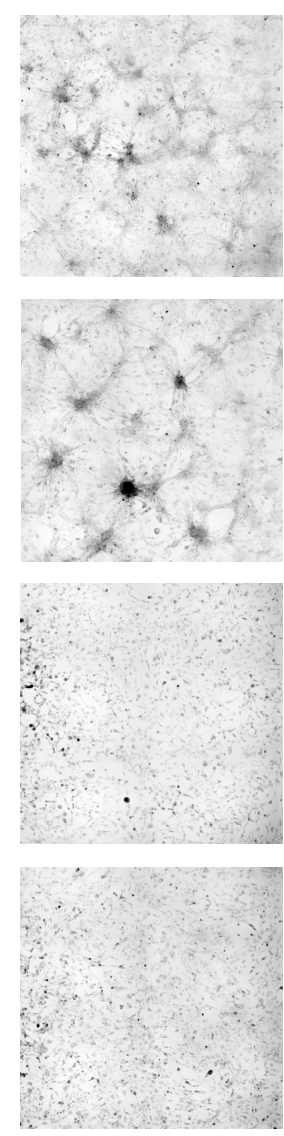

Day 14
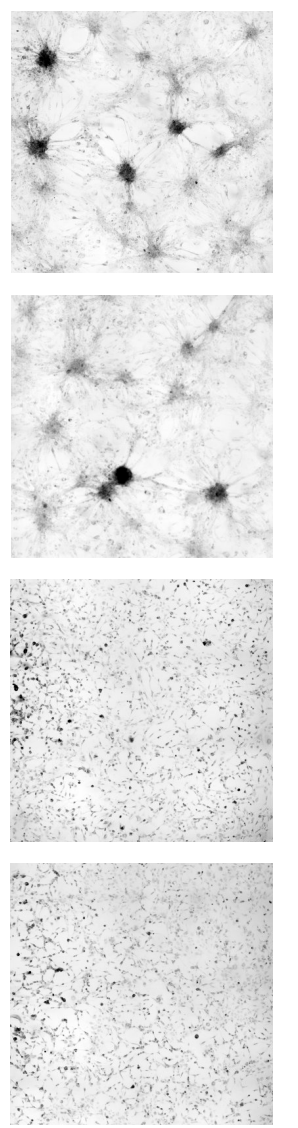

B

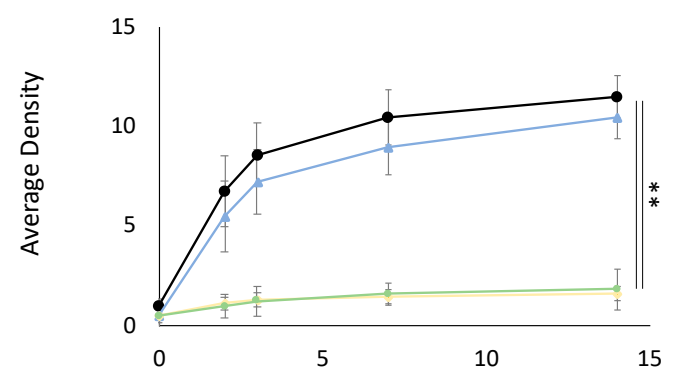

C
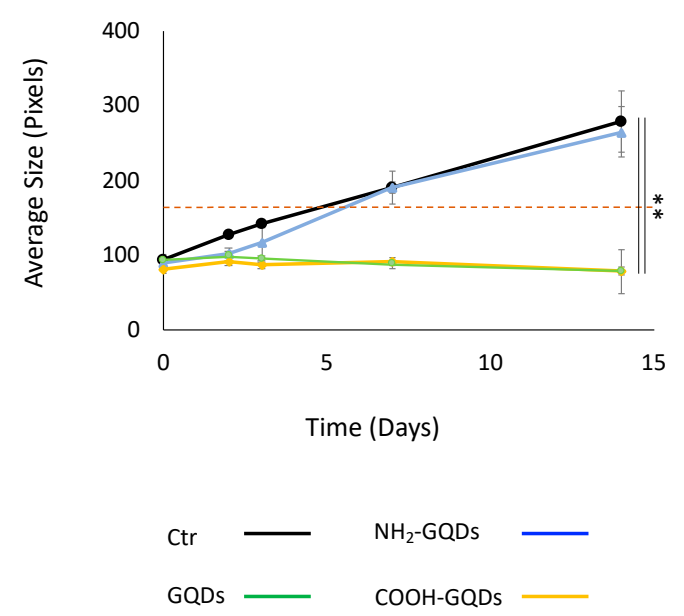

Figure 3. Neurospheres formation. (A) Representative images of neurospheres at 0,7 and 14 days after the administration of $200 \mu \mathrm{g} / \mathrm{mL}$ of GQDs, $\mathrm{NH}_{2}$-GQDs or COOH-GQDs. (B) Average density expressed as number of neurospheres $/ \mathrm{cm}^{2}$, formed in 14 days after the treatment with GQDs. (C) Average size, expressed in pixels, of neurospheres formed in 14 days after the treatment with GQDs. The dashed line represents the value over which the algorithm considered a neurosphere. ${ }^{* *} p<0.01$, one-way ANOVA and Turkey post-hoc test.

\subsection{Membrane Fluidity Shifts in Neurospheres' Formation}

The reason underlying downregulation of neurospheres in GBM could be due to specific cell membrane alterations, which could affect tumor malignancy properties [7]. Proliferation and migration of neural precursor cells are indeed essential determinants of central nervous system development, as well as for the invasiveness of GBM [27]. By tuning membrane fluidity, changes in the stability of integrins, responsible for binding cells to both extracellular matrix and other cell-surface molecules, could disrupt malignancy of GBM. As a fact, a reduction in membrane fluidity has been associated with an increased activity and stability of integrins [1]. Therefore, an increase in the fluidity is associated with a loss of function of these molecules, thus reducing their potential to form clusters. Following this hypothesis, we investigated alterations in GBM cell membrane by means of 
phospholipid phase shifts after the treatment with GQDs. We used Laurdan, a polaritysensitive probe, to measure membrane fluidity. When compared to other methods, polaritysensitive probes show a higher sensitivity for differences in the gel/liquid phases of lipid bilayers [28]. Membrane fluidity quantified by Laurdan is given by the generalized polarization (GP) index, which ranges from -1 (highest membrane fluidity) to +1 (lowest membrane fluidity) [29]. GP values were obtained by confocal microscopy imaging of GBM cells treated with GQDs and labeled with Laurdan (Figure 4A). We measured a GP index of cells treated with $\mathrm{NH}_{2}$-GQDs of $0.17 \pm 0.01$, which had a result similar to that of control untreated cells $(0.19 \pm 0.02$, Figure $4 \mathrm{~B})$. Administration of COOH-GQDs and GQDs caused a significant decrease in membrane fluidity of cells, which shifted to $0.06 \pm 0.03$ and $0.04 \pm 0.02$, respectively (Figure 4B). Interactions between cell membrane and graphene nanosheets have been investigated both theoretically and experimentally. Zhang and Monteiro-Riviere tested the uptake of quantum dots (QDs) with different surface chemistry on human epidermal keratinocytes [30]. They observed a clear uptake of negatively charged COOH-QDs when compared to the positively charged $\mathrm{NH}_{2}-\mathrm{QDs} . \mathrm{NH}_{2}-$ QDs mainly showed an extracellular interaction, while the negative charges of $\mathrm{COOH}$-QDs induced both their uptake and retention at membrane levels. Hanada and colleagues tested QDs with different surface charge in a blood-brain barrier (BBB) transwell model [31]. They demonstrated the capability of both $\mathrm{COOH}-\mathrm{QDs}$ and $\mathrm{NH}_{2}$-QDs to cross the barrier. However, negatively charged quantum dots displayed a longer retention time inside cells, showing a delayed permeability coefficient with respect to amination. Moreover, graphene oxide nanosheets have been theoretically observed to intercalate directly into cell membrane in molecular dynamics simulations by Tu and colleagues, further highlighting the strong dependence of nanoparticles' surface charge on the interaction with cell membrane [32]. We therefore measured the Zeta potential of GQDs. COOH-GQDs revealed a surface charge of $-14 \pm 2.3 \mathrm{mV}$ and GQDs showed a potential of $-24 \pm 1.3 \mathrm{mV}$ (Figure 4C). The strongly negative charge of GQDs was due to the presence of carboxylic, hydroxyl and epoxy nonspecific groups on the surface of the molecule, as depicted by FTIR spectroscopy. $\mathrm{NH}_{2}$-GQDs revealed a less negative surface net charge of $-5 \pm 3.1 \mathrm{mV}$, as a consequence of the presence of positive charges on the amino groups (Figure 4C). Our evidence suggests that negatively charged GQDs increase membrane fluidity, in accordance with literature. We compared the effects of the three GQDs in a 3D scatter plot showing GP index of membranes, Zeta Potential of GQDs and number of neurospheres formed after two weeks (Figure $4 \mathrm{D}$ ). Data are expressed as mean $\pm 95 \%$ confidence interval (CI), to highlight the clear separation in the behavior induced by different GQDs. Changes in membrane fluidity due to surface charge of GQDs indeed resulted in strong correlations with the number of neurospheres formed. COOH-GQDs and GQDs demonstrated a similar effect on GBM cells, increasing membrane fluidity and reducing, at the same time, the number of spheres formed. By calculating the distance between points on the scatter plot, we managed to separate the effect of GQDs into two clear populations (Figure 4D, table). The distance between $\mathrm{NH}_{2}$-GQDs and COOH-GQDs $(\mathrm{D}(3,2))$ on the 3D scatter plot was $20.01 \pm 4.06$ (Figure $4 \mathrm{D}$, table). The distance $\mathrm{D}(3,1)$ between $\mathrm{NH}_{2}$-GQDs and GQDs was $25.83 \pm 4.88$ (Figure $4 \mathrm{D}$, table). The distance between control and COOH-GQDs $(\mathrm{D}(4,2))$ was $24.25 \pm 3.28$ (Figure $4 \mathrm{D}$, table). The distance $\mathrm{D}(4,1)$ between control and GQDs was $30.92 \pm 4.25$ (Figure $4 \mathrm{D}$, table). On the contrary, the distance $\mathrm{D}(2,1)$ between COOH-GQDs and GQDs was significantly smaller, in the range of $95 \% \mathrm{CI}$, resulting in $9.97 \pm 4.45$ (Figure $4 \mathrm{D}$, table). The same happened for the distance $\mathrm{D}(4,3)$, between control and $\mathrm{NH}_{2}$-GQDs, which was $5.38 \pm 3.83$ (Figure $4 \mathrm{D}$, table). Calculating the distance between points in the scatter plot allowed us to separate into two clear populations the effect of GQDs depending on their surface charge, their induced change in membrane fluidity and alteration in the formation of neurospheres. As a fact, amination did not influence membrane fluidity and formation of neurospheres when compared to control cells, resulting in a separate population with respect to $\mathrm{COOH}-\mathrm{GQDs}$ and GQDs. Taken together, these data confirm our starting hypothesis that alterations in membrane fluidity are involved in 
tumor malignancy. We found that negatively charged GQDs-induced increase in membrane fluidity correlated with a significant downregulation of neurospheres formed. This piece of evidence is strongly supported by the literature reporting both a higher interaction between membranes and negative nanoparticles, and a destabilization of cell-to-cell interactions, leading to a reduced clustering of neural precursor cells $[1,22,30]$.

A
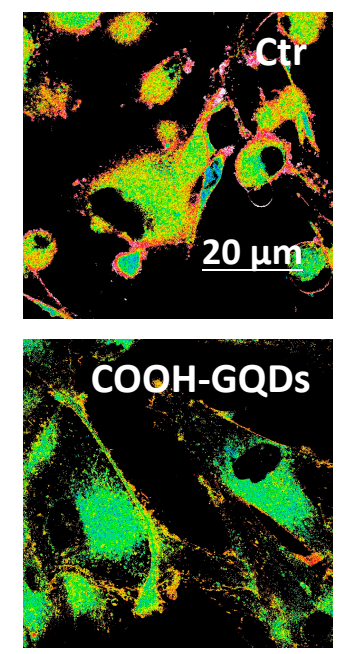

D

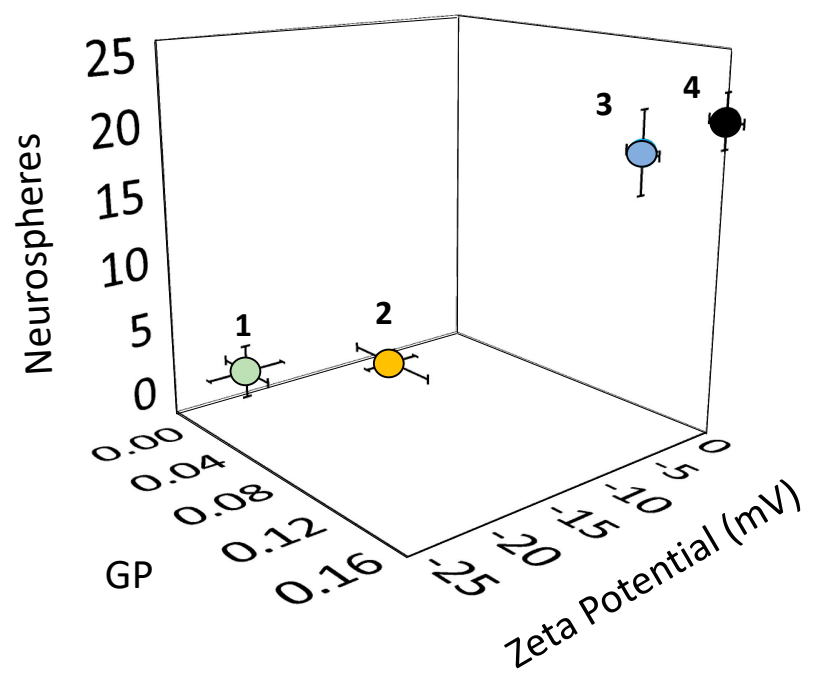

B
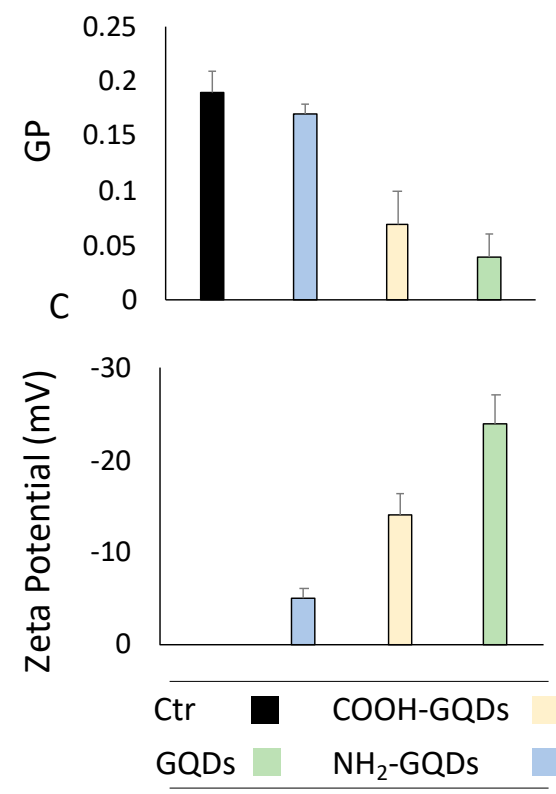

\begin{tabular}{lcc}
\hline & Distance & $\mathbf{\pm}$ \\
\hline D $(\mathbf{3}, \mathbf{2})$ & 20.01 & 4.06 \\
D $(\mathbf{3}, \mathbf{1})$ & 25.83 & 4.88 \\
D $(\mathbf{4}, \mathbf{2})$ & 24.25 & 3.28 \\
D $(\mathbf{4}, \mathbf{1})$ & 30.92 & 4.25 \\
D $(\mathbf{2}, \mathbf{1})$ & 9.97 & 4.45 \\
D $(\mathbf{4}, \mathbf{3})$ & 5.38 & 3.83 \\
\hline
\end{tabular}

Figure 4. Membrane fluidity changes influence neurospheres. (A) Representative Laurdan images of untreated cells and cells treated with the three GQDs. (B) GP values of cells treated with GQDs, extrapolated from analysis of Laurdan images. (C) Surface charge of GQDs. Data are expressed as mean \pm standard deviation. ${ }^{* *} p<0.01$, one-way ANOVA and Turkey post-hoc test. (D) 3D Scatter plot of GP values of cells and surface charge of GQDs correlated with the number of neurospheres formed. Here, data are expressed as mean $\pm 95 \%$ CI The legend is the same as for B and C. The table represents the distance (D) between the four points, along with error propagation.

\subsection{Analysis of the Connections among Neurospheres}

As an astrocytoma, GBM expresses processes similar to endfeet of astrocytes [2]. Astrocytes are mainly known to regulate anatomical integrity of the BBB, as well as for their role in the development of CNS [33]. However, they also respond to neighboring neuronal activity by increasing their cytoplasmic $\mathrm{Ca}^{2+}$ concentration [10]. Such activity is known 
to trigger the release from the astrocytes of signaling molecules (called gliotransmitters, such as glutamate) that may regulate neuronal activity and, importantly, trophism of other neighboring astrocytes [34,35]. Evidence has demonstrated that changes in cytoskeleton of astrocytes occurred when stimuli altered membrane fluidity. These changes in cytoskeleton influenced formation of cell-to-cell interactions between astrocytes, thus altering astrocytes' trophism [36]. Given experimental results involving the effect of GQDs and COOH-GQDs on membrane fluidity, we expected a reduction in the connections formed between GBM neurospheres, accordingly with evidence on astrocytes (Figure 5A).

A

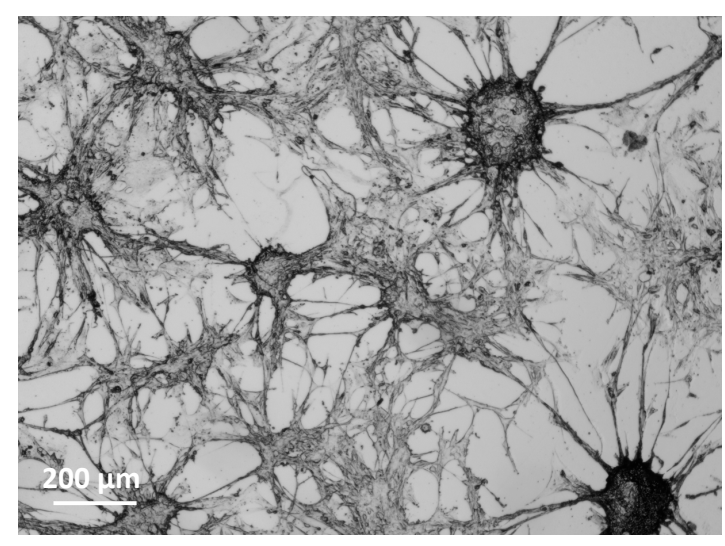

B

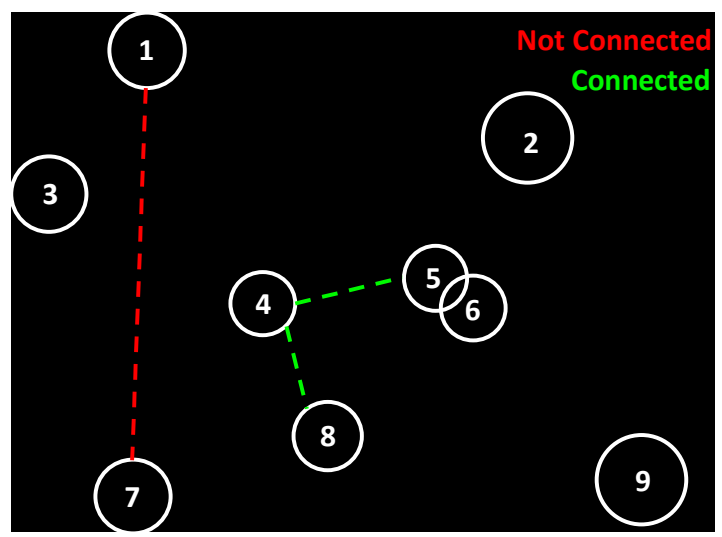

C

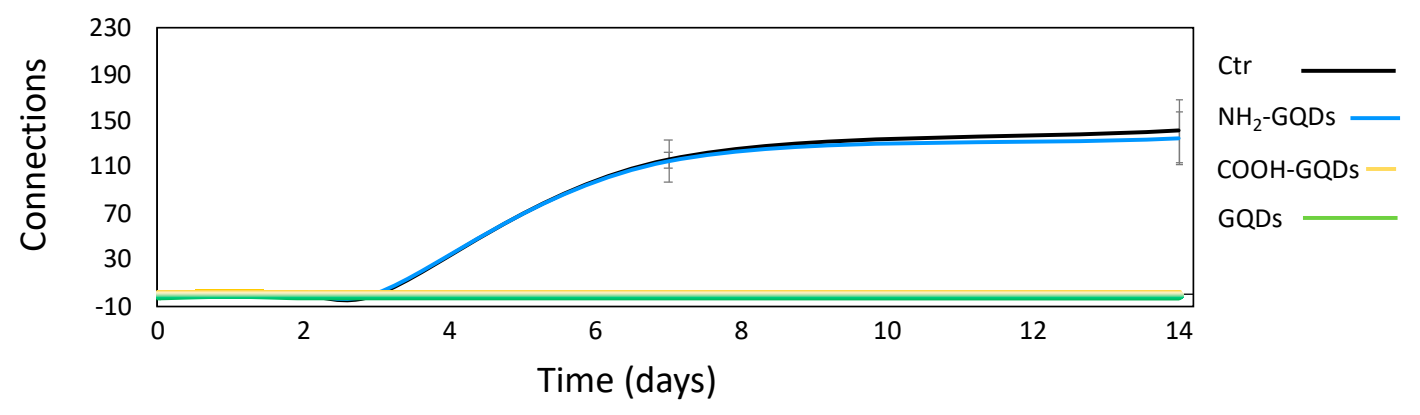

Figure 5. Analysis of connections between neurospheres. (A) Representative image of neurospheres of GBM. (B) Algorithm developed to evaluate the presence of connections between neurospheres and to estimate the number of connections. (C) Measured number of connections after 14 days from the administration of GQDs. Scalebar: $400 \mu \mathrm{m}$.

The spatial arrangement of astrocytes in vivo remains largely unclear. However, it has been pointed out that astrocytes form non-overlapping domains that "tile" the brain space [37]. This suggests a regular spatial arrangement of the cells and leads to a proximitybased coupling rule whereby each astrocyte would be coupled via thigh junctions to its nearest neighbors, at the boundary of their respective non-overlapping domains. To simplify calculations on the number of formed networks, we considered GBM connections as a spatial network. Spatial networks are networks for which the nodes (single neurospheres) are located in a space with a given metric. For most practical cases, the space is the twodimensional space and the metric is the usual Euclidean distance. As a consequence of this definition, the probability of finding a link between two nodes will decrease with the distance [37]. To define the presence of connections, we established a cutoff distance, in pixels, over which two neurospheres were considered as not connected. By applying a binary mask, we isolated single neurospheres, and we were able to consider them as the nodes of the spatial network (Figure 5B). We determined the shortest path between each neurosphere after 14 days of treatment with $200 \mu \mathrm{g} / \mathrm{mL}$ of GQDs, $\mathrm{NH}_{2}-\mathrm{GQDs}$ or COOHGQDs. When the shortest path results were above the cutoff distance, two neurospheres were considered not connected. When the shortest path results were below the cutoff 
distance, two neurospheres were considered connected. $\mathrm{NH}_{2}$-GQDs did not influence GBM connections, consistent with our previous results. In particular, after two weeks, control cells reached $141 \pm 27$ connections, and cells treated with $\mathrm{NH}_{2}$-GQDs reached $135 \pm 22$ connections (Figure 5C). Interestingly, the number of connections followed the same sigmoidal trend of the number of neurospheres formed, suggesting a correlation with spatial confluence of cells after 1 week. Importantly, COOH-GQDs and GQDs, on the other hand, did not allow formation of neurospheres, strongly inhibiting GBM clusterization (Figure 5C).

Our data strongly suggest that GQDs and $\mathrm{COOH}-\mathrm{GQDs}$ alter capability of cancerinitiating stem cells to form neurospheres, indicating a consequent reduction in GBM malignancy. GQDs could act as a powerful tool to mitigate the tumorigenic process in the CNS and to develop therapies targeted to these cells.

\section{Conclusions}

In this study, we have proven that the diverse functionalization of the surface chemistry of GQDs exerts significantly different effects on GBM. Here, a three-dimensional model was used, in which cells tend to cluster in semi-suspension while forming neurospheres. All GQDs demonstrated good biocompatibility even after long time exposure to all the concentrations of GQDs (50,100 and $200 \mu \mathrm{g} / \mathrm{mL}$ ). Growth of neurospheres was monitored for two weeks with or without the administration of GQDs, $\mathrm{NH}_{2}$-GQDs or $\mathrm{COOH}-\mathrm{GQDs}$. $\mathrm{NH}_{2}$-GQDs did not affect GBM spheres' growth. A significant reduction in the formation of novel spheres was measured after the treatment with $200 \mu \mathrm{g} / \mathrm{mL}$ of $\mathrm{COOH}-G Q D$ s and GQDs. This reduction was detected in terms of both number and size of the neurospheres. Importantly, the capability of forming neurospheres depends on a subpopulation of tumor stem cells that have been classified as neurosphere-initiating cells. Proliferation and clustering of neural precursor cells are influenced by their relative membrane stability. Membrane fluidity has been associated with stability of cell-to-cell interactions. A significant increase in membrane fluidity of GBM was measured after the treatment with $\mathrm{COOH}-\mathrm{GQD}$ and GQDs. Augmented fluidity was correlated with the surface net charge of GQDs. It was found that negatively surface-charged GQDs destabilize cell membrane and reduce formation of neurospheres, consequently modulating tumor malignancy. Importantly, another parameter that was affected by GQDs and COOH-GQDs' interaction with GBM is the number of connections between single neurospheres. As for astrocytes, the number of connections is relevant for their trophism and physiology. A clear inhibition of connections between neurospheres was observed after the treatment with $\mathrm{COOH}-G Q D s$ and GQDs, by analyzing spheres as spatial networks.

Taken together, our data strongly indicate the reduction of malignancy of GBM after the treatment with GQDs in a surface-chemistry-dependent manner. These graphene nanoparticles could act as a tool to modulate tumorigenesis, not only in vitro, but also in vivo. The modulation of tumor malignancy mediated by biocompatible GQDs could allow development of target therapies to these stem neurosphere-initiating cells in future clinical translation.

\subsection{Characterization of GQDs}

$\mathrm{COOH}-\mathrm{GQDs}$ and $\mathrm{NH}_{2}$-GQDs were purchased from ACS Material. GQDs were purchased from Sigma-Aldrich. GQDs, $\mathrm{COOH}$-GQDs and $\mathrm{NH}_{2}$-GQDs were optically and microscopically characterized, as previously reported [6,7]. TEM measurements were performed with a Zeiss Libra 120 Transmission electron microscope (Oberkochen, Germany). For Electron microscopy, $50 \mu \mathrm{g} / \mathrm{mL}$ of GQDs was spotted on a TEM tab and let dry at room temperature.

Surface chemical analysis of GQDs was performed using ATR-FTIR by Spectrum One spectrometer (Perkin Elmer). The material under investigation was laid upon the ATR crystal and the spectra were recorded in the wave number range of $4.000-550 \mathrm{~cm}^{-1}$. 
Fluorescence spectra were acquired by using a Cytation 3 Cell Imaging Multi-Mode Reader (Biotek, Winooski, VT, USA) exciting from 260 to $600 \mathrm{~nm}$ and reading the emission from 300 to $700 \mathrm{~nm}$.

Zeta Potential was performed with Zetasizer Nano ZS (Malvern, Worcestershire, UK), equipped with a $633 \mathrm{~nm} \mathrm{He}-\mathrm{Ne}$ laser and operating at an angle of $173^{\circ}$. UV-transparent cuvettes (Malvern, Worcestershire, UK) have been used for experiments with a sample volume of $500 \mu \mathrm{L}$ and a concentration of $100 \mu \mathrm{g} / \mathrm{mL}$. The measurements were performed at a fixed position $(4.65 \mathrm{~mm})$ with an automatic attenuator. Data analysis was performed by Malvern Zetasizer software.

\subsection{Cell Culture}

U87MG glioblastoma cells were purchased from the American Type Culture Collection, (ATTC, Toyota, Japan). Cells were maintained in Dulbecco's modified Eagle's medium (Sigma-Aldrich, St. Louis, MO, USA) supplemented with 10\% fetal bovine serum (FBS, EuroClone), 2\% penicillin-streptomycin (Sigma-Aldrich, St. Louis, MO, USA) and 2\% L-glutamine (Sigma-Aldrich, St. Louis, MO, USA). Cells were cultivated in T75 flasks and kept at $37^{\circ} \mathrm{C}$ in $5 \% \mathrm{CO}_{2}$ humidity.

\subsection{Cell Viability Measurements}

Human GBM cells were cultured on 96-well plates, flat bottom (Corning), at a concentration of $1 \times 10^{4}$ cells/well, with a volume of $100 \mu \mathrm{L}$. Cells were incubated at $37^{\circ} \mathrm{C}$. Then, $24 \mathrm{~h}$ after seeding, cells were treated with GQDs at different concentrations: 200, 100 and $50 \mu \mathrm{g} / \mathrm{mL}$, and incubated at $37{ }^{\circ} \mathrm{C}$ in $5 \% \mathrm{CO}_{2}$. Several 96-wells were cultured for different days after administration of GQDs: after 1, 2, 3, 7 and 14 days, to ensure a triplicate for each measurement. Medium was refilled every 3 days in each well with fresh culture medium containing GQDs to reach starting concentrations. After specific timepoints, the medium was replaced in single 96-wells with fresh culture medium $(100 \mu \mathrm{L})$, and $10 \mu \mathrm{L}$ of 3-(4,5-dimethylthiazol-2-yl)-2,5-diphenyltetrazolium bromide (MTT, Invitrogen) at a concentration of $12 \mathrm{mM}$ was added. After $4 \mathrm{~h}$ of incubation, $100 \mu \mathrm{L}$ of sodium dodecyl sulfate (SDS, Invitrogen), dissolved in $0.01 \mathrm{M} \mathrm{HCl}$, was added to each well, and incubated at $37^{\circ} \mathrm{C}$ in $5 \% \mathrm{CO}_{2}$ for $16 \mathrm{~h}$. Absorbance was read at $570 \mathrm{~nm}$, and data were normalized by control (untreated) cells.

\subsection{Neurospheres Monitoring}

For the formation of neurospheres, human GBM cells were cultured on 24-well plates, flat bottom (Corning), at a concentration of $1 \times 10^{4}$ cells/well, with a volume of $500 \mu \mathrm{L}$. Cells were incubated at $37^{\circ} \mathrm{C}$ in $5 \% \mathrm{CO}_{2}$ humidity. Then, $24 \mathrm{~h}$ after seeding, cells were treated with GQDs at the concentration of $200 \mu \mathrm{g} / \mathrm{mL}$ and further incubated at $37^{\circ} \mathrm{C}$ in $5 \% \mathrm{CO}_{2}$. Medium was refilled every three days in each well with fresh culture medium containing GQDs to reach the final starting concentration of $200 \mu \mathrm{g} / \mathrm{mL}$. Neurospheres' formation was regularly monitored by imaging for 14 days at $4 x$ magnification with Cytation3 Cell Imaging Multi-Mode Reader (Biotek), performing auto-correction of the white balance for each well. Size analysis was carried out with ImageJ software [38]. Briefly, images were converted to 8-bit. A mask was created, and spheres with a circularity of at least 0.3 and a size in pixels $>150$ were considered as neurospheres. The average number of spheres formed was reported this way, along with their average size, expressed in pixels.

\subsection{Membrane Fluidity Measurements}

To perform confocal microscope analysis, U87MG glioblastoma cells were plated on sterile chamber slides (Ibidi) at a concentration of $1 \times 10^{6}$ cell per $\mathrm{mL}$ and then incubated at $37^{\circ} \mathrm{C}$. After $24 \mathrm{~h}$ of incubation, GQDs were administered to cells at the concentration of $200 \mu \mathrm{g} / \mathrm{mL}$ for a further $24 \mathrm{~h}$. For 6-dodecanoyl-2-dimethylamino-naphthalene (Laurdan, Sigma-Aldrich) measurements, GQDs were carefully washed away with PBS, and cells were resuspended in fresh medium containing Laurdan diluted 1:1000 in DMEM, from a 
$2 \mathrm{mM}$ stock solution in dimethyl sulfoxide. Confocal microscopy was carried out using an inverted microscope (Nikon $\mathrm{A} 1 \mathrm{MP}+$, Nikon) equipped with a $60 \times$ oil immersion objective. Images were acquired at $37^{\circ} \mathrm{C}$ for all measurements. For Laurdan excitation, cells were imaged with a wavelength of $400 \mathrm{~nm}$. Laurdan intensity images were recorded simultaneously with emissions in the ranges of 425-475 nm (gel-phase) and 500-550 nm (liquid-phase). To quantify the images collected in this way, Fiji (ImageJ) software was used [38]. The intensities of the two different channels were calculated, and the GP index was calculated [39].

\subsection{Number of Connections among Neurospheres}

To measure the number of connections among neurospheres, images previously acquired were analyzed. As previously described, imaging for 14 days at $4 \mathrm{x}$ magnification with Cytation3 Cell Imaging Multi-Mode Reader (Biotek) was carried out, performing auto-correction of the white balance for each well. Spheres' analysis was carried out with ImageJ software. Images were converted to 8-bit. A mask was created, then a centroid of each sphere with a circularity of at least 0.3 and a size in pixels of at least 200 was identified. In this way, neurospheres were treated like nodes of a random spatial network. Connections among nodes were measured by imposing a cutoff distance (130 pixels) over which two spheres were considered not connected. The shortest path between two centroids was plotted.

\subsection{Statistical Analysis}

Statistical analysis was performed using one-way ANOVA, followed by Bonferroni's post-hoc test. Differences were considered significant when $p<0.01$.

Author Contributions: G.P. performed experimental investigation of cell viability, GQDs characterization, confocal microscopy analysis, developed the algorithm aimed at investigating neurospheres' connections and contributed to article writing. V.P., G.C. and M.P. designed the experiments and methodology and revised the manuscript. A.P. and J.G. performed FTIR characterization. M.D.S. contributed to article writing and revision. All authors have read and agreed to the published version of the manuscript.

Funding: The research leading to these results has received funding from AIRC under IG 2019-ID. 23,124 project-P.I. Massimiliano Papi.

Data Availability Statement: The data presented in this study are available on request from the first author or the corresponding author. The data are not publicly available since they do not represent a database.

Conflicts of Interest: The authors declare that they have no known competing financial interests or personal relationships that could have appeared to influence the work reported in this paper.

\section{References}

1. Gopalakrishna, P.; Chaubey, S.; Manogaran, P.; Pande, G. Modulation of $\alpha 5 \beta 1$ integrin functions by the phospholipid and cholesterol contents of cell membranes. J. Cell. Biochem. 2000, 77, 517-528. [CrossRef]

2. Holland, E.C. Glioblastoma multiforme: The terminator. Proc. Natl. Acad. Sci. USA 2000, 97, 6242-6244. [CrossRef]

3. Ferrara, N.; Hillan, K.J.; Gerber, H.-P.; Novotny, W. Discovery and development of bevacizumab, an anti-VEGF antibody for treating cancer. Nat. Rev. Drug Discov. 2004, 3, 391-400. [CrossRef]

4. Rubenstein, J.L.; Kim, J.; Ozawa, T.; Zhang, M.; Westphal, M.; Deen, D.F.; Shuman, M.A. Anti-VEGF antibody treatment of glio-blastoma prolongs survival but results in increased vascular cooption. Neoplasia 2000, 2, 306-314. [CrossRef]

5. Eampie, L.; Woolf, E.C.; Dardis, C. Immunotherapeutic Advancements for Glioblastoma. Front. Oncol. 2015, 5, 12. [CrossRef]

6. Perini, G.; Palmieri, V.; Ciasca, G.; D’Ascenzo, M.; Primiano, A.; Gervasoni, J.; De Maio, F.; De Spirito, M.; Papi, M. Enhanced Chemotherapy for Glioblastoma Multiforme Mediated by Functionalized Graphene Quantum Dots. Matererials 2020, 13, 4139. [CrossRef]

7. Perini, G.; Palmieri, V.; Ciasca, G.; D'Ascenzo, M.; Gervasoni, J.; Primiano, A.; Rinaldi, M.; Fioretti, D.; Prampolini, C.; Tiberio, F.; et al . Graphene Quantum Dots' Surface Chemistry Modulates the Sensitivity of Glioblastoma Cells to Chemotherapeutics. Int. J. Mol. Sci. 2020, 21, 6301. [CrossRef]

8. Perini, G.; Palmieri, V.; Ciasca, G.; De Spirito, M.; Papi, M. Unravelling the Potential of Graphene Quantum Dots in Biomedicine and Neuroscience. Int. J. Mol. Sci. 2020, 21, 3712. [CrossRef] 
9. Mihaliak, A.M.; Gilbert, C.A.; Li, L.; Daou, M.-C.; Moser, R.P.; Reeves, A.; Cochran, B.H.; Ross, A.H. Clinically relevant doses of chem-otherapy agents reversibly block formation of glioblastoma neurospheres. Cancer Lett. 2010, 296, 168-177. [CrossRef]

10. Lallouette, J.; De Pittà, M.; Ben-Jacob, E.; Berry, H. Sparse short-distance connections enhance calcium wave propagation in a 3D model of astrocyte networks. Front Comput. Neurosci. 2014, 8, 45. [CrossRef]

11. Bez, A.; Corsini, E.; Curti, D.; Biggiogera, M.; Colombo, A.; Nicosia, R.F.; Pagano, S.F.; Parati, E.A. Neurosphere and neuro-sphereforming cells: Morphological and ultrastructural characterization. Brain Res. 2003, 993, 18-29. [CrossRef]

12. Chhabra, V.A.; Kaur, R.; Kumar, N.; Deep, A.; Rajesh, C.; Kim, K.-H. Synthesis and spectroscopic studies of functionalized graphene quantum dots with diverse fluorescence characteristics. RSC Adv. 2018, 8, 11446-11454. [CrossRef]

13. Yang, T.; Liu, L.-H.; Liu, J.-W.; Chen, M.-L.; Wang, J.-H. Cyanobacterium metallothionein decorated graphene oxide nanosheets for highly selective adsorption of ultra-trace cadmium. J. Mater. Chem. 2012, 22, 21909-21916. [CrossRef]

14. Xu, L.; Dai, Y.; Wang, Z.; Zhao, J.; Li, F.; White, J.C.; Xing, B. Graphene quantum dots in alveolar macrophage: Uptake-exocytosis, accumulation in nuclei, nuclear responses and DNA cleavage. Part. Fibre Toxicol. 2018, 15, 1-17. [CrossRef]

15. Yuan, X.; Liu, Z.; Guo, Z.; Ji, Y.; Jin, M.; Wang, X. Cellular distribution and cytotoxicity of graphene quantum dots with different functional groups. Nanoscale Res. Lett. 2014, 9, 108. [CrossRef] [PubMed]

16. Wang, L.; Yin, Y.; Jain, A.; Zhou, H.S. Aqueous Phase Synthesis of Highly Luminescent, Nitrogen-Doped Carbon Dots and Their Application as Bioimaging Agents. Langmuir 2014, 30, 14270-14275. [CrossRef] [PubMed]

17. Fasbender, S.; Allani, S.; Wimmenauer, C.; Cadeddu, R.-P.; Raba, K.; Fischer, J.C.; Bulat, B.; Luysberg, M.; Seidel, C.A.; Heinzel, T.; et al. Uptake dynamics of graphene quantum dots into primary human blood cells following in vitro exposure. RSC Adv. 2017, 7, 12208-12216. [CrossRef]

18. Shang, W.; Zhang, X.; Zhang, M.; Fan, Z.; Sun, Y.; Han, M.; Fan, L. The uptake mechanism and biocompatibility of graphene quantum dots with human neural stem cells. Nanoscale 2014, 6, 5799-5806. [CrossRef] [PubMed]

19. Nurunnabi; Khatun, Z.; Huh, K.M.; Park, S.Y.; Lee, D.Y.; Cho, K.J.; Lee, Y.-K. In Vivo Biodistribution and Toxicology of Carboxylated Graphene Quantum Dots. ACS Nano 2013, 7, 6858-6867. [CrossRef] [PubMed]

20. Li, P.; Xu, T.; Wu, S.; Lei, L.; He, D. Chronic exposure to graphene-based nanomaterials induces behavioral deficits and neural damage inCaenorhabditis elegans. J. Appl. Toxicol. 2017, 37, 1140-1150. [CrossRef]

21. Stupp, R.; Hegi, M.E.; Mason, W.P.; Bent, M.J.V.D.; Taphoorn, M.J.B.; Janzer, R.C.; Ludwin, S.K.; Allgeier, A.; Fisher, B.; Belanger, K.; et al. Effects of radiotherapy with concomitant and adjuvant temozolomide versus radiotherapy alone on survival in glioblastoma in a randomised phase III study: 5-year analysis of the EORTC-NCIC trial. Lancet Oncol. 2009, 10, 459-466. [CrossRef]

22. Gritti, A.; Parati, E.; Cova, L.; Frolichsthal, P.; Galli, R.; Wanke, E.; Faravelli, L.; Morassutti, D.; Roisen, F.; Nickel, D.; et al. Multipotential stem cells from the adult mouse brain proliferate and self-renew in response to basic fibroblast growth factor. J. Neurosci. 1996, 16, 1091-1100. [CrossRef] [PubMed]

23. Miraglia, S.; Godfrey, W.; Yin, A.H.; Atkins, K.; Warnke, R.; Holden, J.T.; Bray, R.A.; Waller, E.K.; Buck, D.W. A novel fivetransmembrane hematopoietic stem cell antigen: Isolation, characterization, and molecular cloning. Blood 1997, 90, 5013-5021. [CrossRef] [PubMed]

24. Doetsch, F. The glial identity of neural stem cells. Nat. Neurosci. 2003, 6, 1127-1134. [CrossRef] [PubMed]

25. Jung, C.S.; Foerch, C.; Schanzer, A.; Heck, A.; Plate, K.H.; Seifert, V.; Steinmetz, H.; Raabe, A.; Sitzer, M. Serum GFAP is a diagnostic marker for glioblastoma multiforme. Brain 2007, 130, 3336-3341. [CrossRef]

26. Juzenas, P.; Steponkiene, S.; Kavaliauskiene, S.; Purviniene, R.; Rotomskis, R. Quantum dots affect expression of CD133 surface antigen in melanoma cells. Int. J. Nanomed. 2011, 6, 2437-2444. [CrossRef] [PubMed]

27. Jacques, T.S.; Relvas, J.B.; Nishimura, S.; Pytela, R.; Edwards, G.M.; Streuli, C.H.; Ffrench-Constant, C. Neural precursor cell chain migration and division are regulated through different beta1 integrins. Development 1998, 125, 3167-3177.

28. Amaro, M.; Reina, F.; Hof, M.; Eggeling, C.; Sezgin, E. Laurdan and Di-4-ANEPPDHQ probe different properties of the mem-brane. J. Phys. D Appl. Phys. 2017, 50, 134004. [CrossRef]

29. Parasassi, T.; De Stasio, G.; d'Ubaldo, A.; Gratton, E. Phase fluctuation in phospholipid membranes revealed by Laurdan fluo-rescence. Biophys. J. 1990, 57, 1179-1186. [CrossRef]

30. Zhang, L.W.; Monteiro-Riviere, N.A. Mechanisms of Quantum Dot Nanoparticle Cellular Uptake. Toxicol. Sci. 2009, 110, 138-155. [CrossRef]

31. Hanada, S.; Fujioka, K.; Inoue, Y.; Kanaya, F.; Manome, Y.; Yamamoto, K. Cell-Based in Vitro Blood-Brain Barrier Model Can Rapidly Evaluate Nanoparticles' Brain Permeability in Association with Particle Size and Surface Modification. Int. J. Mol. Sci. 2014, 15, 1812-1825. [CrossRef] [PubMed]

32. Tu, Y.; Lv, M.; Xiu, P.; Huynh, T.; Zhang, M.; Castelli, M.; Liu, Z.; Huang, Q.; Fan, C.; Fang, H.; et al. Destructive extraction of phospholipids from Escherichia coli membranes by graphene nanosheets. Nat. Nanotechnol. 2013, 8, 594-601. [CrossRef] [PubMed]

33. Goldstein, G.W. Endothelial Cell-Astrocyte Interactions. Ann. N. Y. Acad. Sci. 1988, 529, 31-39. [CrossRef] [PubMed]

34. Chen, Y.; Swanson, R.A. Astrocytes and Brain Injury. Br. J. Pharmacol. 2003, 23, 137-149. [CrossRef]

35. Perini, G.; Ciasca, G.; Minelli, E.; Papi, M.; Palmieri, V.; Maulucci, G.; Nardini, M.; Latina, V.; Corsetti, V.; Florenzano, F.; et al. Dynamic structural determinants underlie the neurotoxicity of the N-terminal tau 26-44 peptide in Alzheimer's disease and other human tauopathies. Int. J. Biol. Macromol. 2019, 141, 278-289. [CrossRef]

36. Zhu, D.; Tan, K.S.; Zhang, X.; Sun, A.Y.; Sun, G.Y.; Lee, J.C.-M. Hydrogen peroxide alters membrane and cytoskeleton properties and increases intercellular connections in astrocytes. J. Cell Sci. 2005, 118, 3695-3703. [CrossRef] 
37. Barthelemy, M. Spatial networks. Phys. Rep. 2011, 499, 1-101. [CrossRef]

38. Moriconi, C.; Palmieri, V.; Di Santo, R.; Tornillo, G.; Papi, M.; Pilkington, G.; De Spirito, M.; Gumbleton, M. INSIDIA: A FIJI Macro Delivering High-Throughput and High-Content Spheroid Invasion Analysis. Biotechnol. J. 2017, 12. [CrossRef]

39. Leo, M.; Di Giacinto, F.; Nardini, M.; Mazzini, A.; Rossi, C.; Porceddu, E.; Papi, M.; Grieco, A.; De Spirito, M.; Ciasca, G. Erythrocyte viscoelastic recovery after liver transplantation in a cirrhotic patient affected by spur cell anaemia. J. Microsc. 2020, 280, 287-296. [CrossRef] 